\title{
BMJ Open Dietary factors and diabetes-related health outcomes in patients with type 2 diabetes: protocol for a systematic review and meta-analysis of prospective observational studies
}

To cite: Barbaresko J,

Neuenschwander M,

Schwingshackl L, et al. Dietary factors and diabetes-related health outcomes in patients with type 2 diabetes: protocol for a systematic review and meta-analysis of prospective observational studies. BMJ Open 2019;9:e027298. doi:10.1136/ bmjopen-2018-027298

- Prepublication history for this paper is available online. To view these files please visit the journal online (http://dx.doi org/10.1136/bmjopen-2018027298).

Received 15 0ctober 2018 Revised 4 April 2019 Accepted 21 June 2019

Check for updates

(c) Author(s) (or their employer(s)) 2019. Re-use permitted under CC BY-NC. No commercial re-use. See rights and permissions. Published by BMJ.

${ }^{1}$ Institute for Biometrics and Epidemiology, German Diabetes Center, Leibniz Center for Diabetes Research at Heinrich Heine University Düsseldorf, Düsseldorf, Germany ${ }^{2}$ Institute for Evidence in

Medicine, Faculty of Medicine and Medical Center, University of Freiburg, Freiburg, Germany

Correspondence to Dr Janett Barbaresko; janett.barbaresko@ddz.de

\section{ABSTRACT}

Introduction Type 2 diabetes (T2D) is a major health concern associated with several comorbidities such as diabetic chronic kidney disease, neuropathy and cardiovascular diseases. Many of these complications may be preventable by an adequate lifestyle, including a favourable dietary behaviour, additionally to pharmacological management. In general, dietary guidelines for patients with diabetes recommend a hypocaloric diet to achieve a normal weight, but there is a lack of detailed instructions on specific nutrients and foods to prevent diabetes-related outcomes. Therefore, the aim of this systematic review and meta-analysis is to summarise the available evidence on the association between dietary factors and health-related outcomes in patients with T2D.

Methods and analysis A systematic literature search will be conducted in PubMed and Web of Science in May 2019 to identify prospective observational studies investigating dietary factors in association with major complications in patients with T2D. We will include studies investigating dietary patterns, food groups, foods, macronutrients and micronutrients as well as secondary plant compounds. As diabetes-related outcomes, we will include macrovascular (cardiovascular and cerebrovascular diseases) and microvascular outcomes (nephropathy, neuropathy and retinopathy), as well as cancer, quality of life, depression, cognitive disorders and mortality. We will conduct doseresponse meta-analyses using random effects models. We will investigate heterogeneity across studies and publication bias. To assess the risk of bias and quality of the included studies, we will use the Cochrane risk of bias tool ROBINS-I and the quality of evidence will be assessed using Grades of Recommendation, Assessment, Development, and Evaluation. Ethics and dissemination As the systematic review is based on published studies, ethical considerations are not required. The systematic review and meta-analysis will be published in a peer-reviewed Journal.

PROSPERO registration number CRD42018110669

\section{INTRODUCTION}

Type 2 diabetes (T2D) is a major health concern with an estimated global prevalence
Strengths and limitations of this study

- The novelty of this systematic review will be the investigation of the current evidence on associations between dietary factors and risk of diabetes complications in individuals with type 2 diabetes (T2D).

- This systematic review will provide a comprehensive overview of dietary patterns, foods and nutrients in association with a range of diabetes-related outcomes: macrovascular (cardiovascular diseases) and microvascular outcomes (nephropathy, neuropathy and retinopathy), cancer, health-related quality of life, depression, cognitive disorders and mortality.

- We will conduct linear and non-linear dose-response meta-analyses to investigate the impact of different dietary factors on the risk of health-related outcomes in patients with T2D.

- Evidence will be derived from prospective observational studies, and thus a conclusive answer on causality cannot be provided.

of $8.8 \%$ in the adult population and the prevalence is still expected to increase. ${ }^{1}$ It is well known that hyperglycaemia is associated with many comorbidities such as diabetic chronic kidney disease, retinopathy, neuropathy, foot ulcers, and cardiovascular diseases (CVD). Thus, patients with diabetes are at an increased risk of premature mortality. According to the International Diabetes Federation, about 4 million adults aged 20-79 years died due to diabetes worldwide in $2017 .^{1}$ Recently, the Global Burden of Disease Study published a report presenting diabetes as one of the major causes of years of life lost among non-communicable diseases. ${ }^{2}$ Thus, management of hyperglycaemia and secondary prevention of comorbidities in patients with diabetes is of high public health interest.

T2D is a metabolic multicausal disorder. Many risk factors for diabetes are known, 
including non-modifiable risk factors, for example, age and genetic predisposition, as well as modifiable risk factors such as an unfavourable diet, overweight, smoking and physical inactivity. ${ }^{3}$ With regard to diet, several dietary recommendations for the prevention of T2D have been proposed. ${ }^{14-7}$ Thus, recommendations include reduced energy intake for weight management and general healthy diet patterns such as a Mediterranean diet, Dietary Approaches to Stop Hypertension (DASH) or plant-based diets.

In contrast, dietary guidelines for patients with T2D to prevent diabetes complications are scarce and mostly not evidence-based. In general, a hypocaloric diet is recommended to achieve a normal weight, ${ }^{8}$ as well as the adherence to dietary patterns like the Mediterranean diet and plant-based diets similar to general recommendations for the prevention of T2D. ${ }^{9}$ Moreover, recommendations refer to surrogate markers such as blood glucose and lipids instead of health-related outcomes. ${ }^{7}$ Specific instructions on nutrients and foods are missing or are based on recommendations for the general population ${ }^{5}$ and thus, specific guidelines on the prevention of diabetes complications are of main interest. So far, individual studies investigated dietary factors in association to different diabetes-related outcomes in patients with T2D. For instance, total carbohydrate intake was not associated with diabetic nephropathy, retinopathy and CVD in a Japanese study with a follow-up of 8 years. ${ }^{10}$ In contrast, higher fruit intake has been shown to reduce the relative risk of diabetic retinopathy by about $50 \%$ compared with the lowest quartile of fruit intake. ${ }^{11}$ Moreover, sodium intake was positively associated with the risk of CVD, but not with all-cause mortality in patients with T2D. ${ }^{12}$ Evidence on the impact of different dietary factors on diabetes complications has not been comprehensively summarised. There are systematic reviews on diet and retinopathy. ${ }^{13-15}$ However, a systematic review and meta-analysis on other health-related outcomes in patients with T2D is missing.

Therefore, this protocol presents the methodology of a systematic review and meta-analysis aiming at summarising and investigating the current literature on the associations of dietary factors and the risk of diabetes complications and health outcomes.

\section{Objectives}

The aims of the systematic review and meta-analysis are

- To summarise the currently available data on dietary factors, including dietary patterns, food groups, foods, macronutrients and micronutrients, secondary plant compounds and the risk of diabetes-related outcomes such as CVD, neuropathy, diabetic foot ulcers, amputations, nephropathy, retinopathy, cancer, health-related quality of life, depression, cognitive disorders and mortality in patients with T2D derived from prospective observational studies; and

- to quantitatively summarise the impact of different types of dietary factors on diabetic complications and mortality in linear and non-linear dose-response meta-analysis.

\section{METHODS}

The present protocol was planned, conducted, and reported according to the Preferred reporting items for systematic review and meta-analysis protocols (PRISMA-P). ${ }^{16}$ The systematic review and meta-analysis was registered in PROSPERO International Prospective Register of Systematic Reviews (registration number: CRD42018110669). Amendments to this protocol will be included into updated versions of the PROSPERO, if needed.

\section{Eligibility criteria}

We will include studies in the systematic review and meta-analysis if the following criteria are met:

1. Participants: We will include patients with T2D aged 18 or older. Studies which solely focused on children, adolescents, participants with pre-diabetes, type 1 diabetes or gestational diabetes will be excluded, as well as animal and cell culture studies.

2. Exposure: Dietary factors including dietary patterns, food groups, foods, macronutrients and micronutrients, and secondary plant compounds (eg, polyphenols) and supplements (eg, vitamin E, magnesium). Studies reporting on dietary factors in combination with other lifestyle factors (eg, physical activity, lifestyle index) will be excluded.

3. Outcome: We will investigate the following diabetes complications: macrovascular complications including fatal and non-fatal cardiovascular diseases, microvascular complications: diabetic neuropathy, diabetic foot ulcers, amputations, diabetic nephropathy, diabetic retinopathy; cancer, health-related quality of life, depression, cognitive disorders and mortality.

4. Study design: We will include prospective observational studies (including cohort, nested case-control, case-cohort studies and follow-up studies of intervention studies) published in a peer-reviewed journal. Cross-sectional, case only or case-control studies, conference abstracts, comments, letters and reviews will be excluded from the systematic review.

Articles written in English or German will be eligible for the systematic review and meta-analysis. Articles in other languages will be excluded.

\section{Exposure}

We will evaluate the impact of different dietary factors assessed by established dietary assessment instruments such as food frequency questionnaires, diet history, 24-hour dietary recalls, dietary records, or biomarkers of dietary intake. We will include the following dietary factors:

- Dietary patterns, dietary habits and diet quality: We will include dietary patterns generated in the primary study and derived by hypothesis-driven methods, namely dietary indices (eg, Healthy Eating 
Index $,{ }^{17}{ }^{18} \mathrm{DASH},{ }^{19}$ dietary scores (eg, Mediterranean Diet Score $^{20}$ ), exploratory-derived dietary patterns by eg principal component analysis, factor analysis or reduced rank regression, dietary habits (eg, vegetarian or vegan diets), and diet quality (eg, glycaemic index or glycaemic load).

- Food groups or foods: We will especially focus on the following food groups: whole-grain products, refined grain products, potatoes, rice, vegetables, legumes, fruit, nuts, eggs, meat (red meat, processed meat, and poultry), dairy products, fish, tea, coffee, sweets, sugar-sweetened beverages, juice and alcoholic beverages.

- Macronutrients (carbohydrates, fats, protein), micronutrients (vitamins, minerals), fibre and secondary plant compounds.

- Dietary supplements: vitamins (eg, vitamin E or D), minerals (eg, magnesium or calcium), or other products (eg, fish oils).

\section{Outcome}

We will include objectively measured outcomes such as CVD, neuropathy, nephropathy, retinopathy, cancer, and mortality assessed through record linkage, death certificates, disease-specific registries, medical records or accepted clinical criteria in the original papers; as well as subjectively measured outcomes such as health-related quality of life, depression and cognitive disorders evaluated by validated or established instruments. In particular, we will focus on the following groups of outcomes:

- Macrovascular outcomes:

- Total CVD, including coronary heart diseases and stroke.

- Coronary heart diseases, including myocardial infarction, acute coronary syndrome, and ischaemic heart disease.

- Heart failure.

- Stroke.

- Microvascular outcomes:

- Diabetic polyneuropathy, including the presence of symptoms and/or signs of peripheral nerve dysfunction in people with diabetes after the exclusion of other causes, diabetic foot ulcers, and amputations.

- Diabetic nephropathy/chronic kidney disease.

- Diabetic retinopathy, including diabetic retinopathy, macular oedema, vitreous haemorrhage, and diabetes-related blindness.

- Cancer: total cancer and site-specific cancer.

- Health-related quality of life, including global health-related quality of life, mental, social, emotional, role and physical functioning, as well as diabetes specific scales for example, problem areas in diabetes and diabetes distress.

- Depression.

- Cognitive disorders, including cognitive decline, cognitive impairment, and dementia (Alzheimer's disease and all-cause dementia).
Mortality: all-cause and cause-specific mortality.

\section{Search strategy to identify relevant studies}

The systematic literature search will be performed in PubMed and Web of Science. The search strategy was developed in PubMed and will be adapted for Web of Science. The systematic literature search will be conducted in May 2019. The full search strategy for PubMed is presented in table 1. Afterwards, we will screen the reference lists of all eligible studies and relevant reviews to identify additional studies meeting our inclusion criteria. The search results will be managed using the citation software Reference Manager V.12.

\section{Study selection process}

The titles and abstracts of all identified studies in the databases will be independently screened by two researchers. In the second step, the full-texts of potentially relevant studies will be retrieved and investigated according the inclusion criteria. The reasons for exclusion of studies will be documented. Any disagreements between the two researchers will be resolved by consensus or by consultation of a third researcher. Furthermore, the reference lists of all included studies will be screened for further potentially relevant articles. If there are multiple publications of the same study population we will include the most recent and comprehensive report including the largest sample size. The study selection process will be presented in a flow chart.

\section{Data extraction}

One reviewer will extract the following information from the identified studies using a predefined data extraction form: the first author's last name, year of publication, country of study conduct, study design, cohort name, sample size, number of cases, sex and age of the patients at study entry, duration of follow-up, type of exposure and assessment method, quantity of dietary factors across categories, type of outcome and assessment, the fully-adjusted risk estimate (Risk Ratio) with corresponding 95\% CI and adjustment factors. A second reviewer will check all information for accuracy. If there is relevant information missing in the articles, we will contact the corresponding authors to obtain the data. We will exclude studies from the meta-analyses if we were unable to obtain relevant data. All contacts with authors will be documented.

\section{Study quality and risk of bias}

To evaluate the study quality of the prospective studies and potential risks of bias, we will use the risk of bias in non-randomised studies of interventions (ROBINS-I) tool. ${ }^{21}$ This tool was proposed by Cochrane and can also be applied to assess the risk of bias in observational studies. The tool is divided into the following domains: (1) bias due to confounding, (2) bias in selection of participants into the study, (3) bias in measurement of the exposure, (4) bias due to misclassification of exposure during follow-up, (5) bias due to missing data, (6) bias in measurement of outcomes, and (7) bias in selection of 


\section{Table 1 Search strategy in PubMed}

\#1 Diet $\quad$ diet [Mesh] OR diet [tiab] OR nutrition [tiab] OR dietary [tiab] OR intake [tiab] OR consumption [tiab] OR food [tiab] OR foods [tiab] OR food [MeSH] OR beverage [tiab] OR beverages [tiab] OR beverages [Mesh] OR drink [tiab] OR drinking [tiab] OR bread [tiab] OR cereals [tiab] OR grains [tiab] OR whole-grain [tiab] OR soy [tiab] OR soya [tiab] OR potatoes [tiab] OR legumes [tiab] OR rice [tiab] OR pasta [tiab] OR vegetables [tiab] OR fruit [tiab] OR milk [tiab] OR dairy [tiab] OR eggs [tiab] OR meat [tiab] OR fish [tiab] OR seafood [tiab] OR nuts [tiab] OR sweets [tiab] OR alcohol [tiab] OR nutrient [tiab] OR nutrients [tiab] OR coffee [tiab] OR tea [tiab] OR juice [tiab] OR macronutrients [tiab] OR fats [tiab] OR "fatty acids" [tiab] OR carbohydrates [tiab] OR fibre [tiab] OR fiber [tiab] OR sugar [tiab] OR protein [tiab] OR micronutrients [tiab] OR vitamin [tiab] OR vitamins [tiab] OR mineral [tiab] OR minerals [tiab] OR polyphenols [tiab]

\begin{tabular}{|c|c|c|}
\hline \#2 & Diabetes & diabetes [tiab] OR “Diabetes Mellitus, Type 2" [Mesh] OR diabetic [tiab] \\
\hline \#3 & CVD & $\begin{array}{l}\text { macrovascular [tiab] OR "cardiovascular disease" [tiab] OR "cardiovascular diseases" [tiab] OR } \\
\text { CVD [tiab] OR "heart disease" [tiab] OR "myocardial infarction" [tiab] OR "heart failure" [tiab] OR } \\
\text { "cerebrovascular diseases" [tiab] OR stroke [tiab] }\end{array}$ \\
\hline \#4 & $\begin{array}{l}\text { Microvascular } \\
\text { complications }\end{array}$ & $\begin{array}{l}\text { microvascular [tiab] OR retinopathy [tiab] OR neuropathy [tiab] OR "foot ulcer" [tiab] OR } \\
\text { amputations [tiab] OR nephropathy [tiab] OR "kidney disease" [tiab] OR "renal disease" [tiab] }\end{array}$ \\
\hline \#5 & Cancer & cancer [tiab] OR neoplasm [tiab] OR neoplasms [tiab] OR tumor [tiab] OR tumour [tiab] \\
\hline \#7 & $\begin{array}{l}\text { Health-related } \\
\text { quality of life and } \\
\text { depression }\end{array}$ & "health-related quality of life" [tiab] OR depression [tiab] OR depressive [tiab] \\
\hline \#8 & Mortality & mortality [tiab] OR death [tiab] OR survival [tiab] \\
\hline \#11 & & \#1 AND \#2 AND \#10 AND \#9 \\
\hline
\end{tabular}

reported results. Signalling questions within each domain facilitate the judgement of potential risk of bias. A study will be considered as being at low risk of bias if the study has been rated to be at low risk of bias in all domains; low to moderate risk of bias if the study has been judged to be probably at risk for one domain; serious risk of bias if the study has been rated as high risk for more than one domain, and critical risk of bias if a study is judged to be at critical risk in at least one domain. If relevant information in at least one domain are missing, the study will be classified as no information. Following the assessment of risk of bias, the body of evidence for each exposure and outcome association will be rated using the Grades of Recommendation, Assessment, Development, and Evaluation (GRADE) approach. ${ }^{22}$ This method takes into account the within-study risk of bias, inconsistency, indirectness and imprecision between the studies, publication bias, large magnitude of effect and dose-response relationship. Two reviewers will independently assess the risk of bias of all included studies and rate the certainty of evidence. Any disagreements between the two investigators will be resolved by consensus or consultation of a third researcher.

\section{Data analysis and presentation}

Data analyses will be performed using the statistical software Stata V.14 (Statacorp). We will conduct meta-analyses using a random effects model when two or more studies on the same exposure and outcome are available by applying the method provided by DerSimonian and Laird. ${ }^{23}$ We will separately investigate studies on dietary intake, dietary supplements, and biomarkers of dietary intake. If a study reported only separate risk estimates for men and women, we will pool the data using a fixed effect model before entering the study in the overall meta-analysis. We will focus on dose-response meta-analyses and high versus low meta-analyses will only be conducted if information on dose-response associations are lacking. We will conduct linear dose-response meta-analyses using the method as described by Greenland and Longnecker. ${ }^{24}$ In addition, we will explore the shape of the association by conducting non-linear dose-response meta-analysis as described by Orsini et al. ${ }^{25}$ This method requires the following data for at least three exposure categories: the quantified exposure value (eg, grams per day), the effect estimate with the corresponding $95 \%$ CI, and the number of cases and person-years. If the number of cases in single categories has not been reported in one study, but information on the total number of cases and total personyears or the number of total patients plus follow-up period have been provided, the number of cases will be equally distributed across the quantiles, and for specified categories as described previously. ${ }^{26}$ If a range of dietary intake 
will be presented, the midpoint value will be assigned as exposure level for the respective category. To investigate heterogeneity, we will calculate tau-squared to assess the between-study variance and $I^{2}$ statistic to investigate the variability of the observed effects in the meta-analyses. ${ }^{27}$ We will also calculate prediction intervals to present how widely the single study effects vary across the studies. ${ }^{28}$ We will conduct subgroup analyses and meta-regression to investigate possible sources of heterogeneity across studies such as sex, age, study length, study origin, duration of diabetes, medical treatment, if sufficient data are available. Moreover, potential publication bias will be investigated visually using funnel plots and Egger's test, whereas a $\mathrm{p}$ value $<0.1$ indicates potential publication bias.

The results of the systematic review and meta-analyses will be reported according to the Meta-analysis Of Observational Studies in Epidemiology (MOOSE) ${ }^{29}$ The characteristics and results of all studies will be presented in summary tables and forest plots where appropriate.

\section{Patient and public involvement}

We considered the opinion of patients with diabetes mellitus regarding the research question and outcome selection. In a previous project, partly conducted at our institution, patients' interests, information needs and preferences concerning diabetes research was assessed. The study was conducted in 26 adults with diabetes from North Rhine-Westphalia, Germany. The patients were interviewed in moderated focus groups and transcripts were analysed in a multidisciplinary team using qualitative content analysis. ${ }^{30}$ Patients with diabetes expressed their interests, among others, in accessibility to comprehensible knowledge regarding lifestyle recommendations, including dietary advices. In particular, they asked about different effects of dietary patterns containing different amounts of carbohydrates and fats. Moreover, patients also discussed about complications due to diabetes. One central point was the prevention of complications, especially cardiovascular diseases, polyneuropathy, diabetic foot pain and ulcer, nephropathy and retinopathy. Additionally, health-related quality of life and its predictors were mentioned as an important outcome regarding diabetes research. These findings underline that patients with diabetes are searching for clear and comprehensible information on dietary recommendations with regard to prevention of diabetes complications and health-related quality of life.

Moreover, based on this survey, ${ }^{30}$ a questionnaire was developed and tested in a pretest. At the end of 2018, the questionnaire had been sent out to a representative study sample, including 3000 patients with diabetes registered at a local health insurance. These findings will help us to focus on (further) patient-relevant outcomes, which we can incorporate in extensions of our systematic review and meta-analysis.

\section{CONCLUSION}

To our knowledge, this systematic review and meta-analysis is the first to comprehensively summarise the available evidence and conduct meta-analyses on the association of dietary factors and diabetes-related outcomes in patients with T2D. We will include studies investigating dietary patterns, food groups or single foods, macronutrients and micronutrients, or secondary plant compounds (polyphenols) in association with CVD, neuropathy, diabetic foot ulcers, pain and amputations, nephropathy, retinopathy, cancer, quality of life, depression, cognitive disorders and mortality in individuals with T2D.

As diabetes management is important to prevent comorbidities and dietary guidelines for patients with T2D are scarce, this systematic review will provide important impact regarding evidence-based recommendations on dietary behaviour for patients with prevalent diabetes. Further research questions can be developed, for example, dietary factors in association to further endpoints.

Contributors JB, LS and SS designed the study question and developed the search term of the systematic review and meta-analysis. JB and SS drafted the present protocol. JB and MN will conduct the systematic literature search and will be involved in data acquisition. JB and LS will conduct the assessment of risk of bias. $\mathrm{JB}$ and SS will conduct the analyses. JB, MN, LS and SS will interpret the results. $\mathrm{JB}, \mathrm{MN}, \mathrm{LS}$ and SS critically reviewed the protocol and approved submission of the final manuscript.

Funding The German Diabetes Center is funded by the German Federal Ministry of Health and the Ministry of Innovation, Science and Research of the State of North Rhine-Westphalia. This work is funded as part of a project investigating diabetes complications (Sondertatbestand - "Begleit- und Folgeerkrankungen des Diabetes").

\section{Competing interests None declared.}

Patient consent for publication Not required.

Ethics approval As the systematic review is based on published studies, ethical considerations are not required. This systematic review and meta-analysis will be published in a peer-reviewed journal and will be presented at scientific conferences.

Provenance and peer review Not commissioned; externally peer reviewed.

Open access This is an open access article distributed in accordance with the Creative Commons Attribution Non Commercial (CC BY-NC 4.0) license, which permits others to distribute, remix, adapt, build upon this work non-commercially, and license their derivative works on different terms, provided the original work is properly cited, appropriate credit is given, any changes made indicated, and the use is non-commercial. See: http://creativecommons.org/licenses/by-nc/4.0/.

\section{REFERENCES}

1. International Diabetes Federation. IDF diabetes atlas. 8th edn. Brussels, Belgium: International Diabetes Federation, 2017.

2. GBD 2016 Causes of Death Collaborators. Global, regional, and national age-sex specific mortality for 264 causes of death, 19802016: a systematic analysis for the Global Burden of Disease Study 2016. Lancet 2017;390:1151-210.

3. Bellou V, Belbasis L, Tzoulaki I, et al. Risk factors for type 2 diabetes mellitus: an exposure-wide umbrella review of meta-analyses. PLOS One 2018;13:e0194127.

4. Paulweber B, Valensi P, Lindström J, et al. A European evidencebased guideline for the prevention of type 2 diabetes. Horm Metab Res 2010;42(Suppl 1):S3-36.

5. Ley $\mathrm{SH}$, Hamdy O, Mohan V, et al. Prevention and management of type 2 diabetes: dietary components and nutritional strategies. Lancet 2014;383:1999-2007. 
6. American Diabetes Association. 5. prevention or delay of type 2 diabetes: Standards of Medical Care in Diabetes-2018. Diabetes Care 2018;41(Suppl 1):S51-S54.

7. Dyson PA, Twenefour D, Breen C, et al. Diabetes UK evidence-based nutrition guidelines for the prevention and management of diabetes. Diabet Med 2018;35:541-7.

8. International Diabetes Federation. Recommendations for managing type 2 diabetes in primary care: international diabetes federation. 2017. www.idf.org/managing-type2-diabetes

9. American Diabetes Association. 4. lifestyle management: Standards of Medical Care in Diabetes-2018. Diabetes Care 2018;41(Suppl 1):S38-S50.

10. Horikawa C, Yoshimura Y, Kamada C, et al. Is the proportion of carbohydrate intake associated with the incidence of diabetes complications?-an analysis of the japan diabetes complications study. Nutrients 2017;9:pii:E113.

11. Tanaka S, Yoshimura Y, Kawasaki R, et al. Fruit intake and incident diabetic retinopathy with type 2 diabetes. Epidemiology 2013;24:204-11.

12. Horikawa C, Yoshimura $Y$, Kamada C, et al. Dietary sodium intake and incidence of diabetes complications in Japanese patients with type 2 diabetes: analysis of the Japan Diabetes Complications Study (JDCS). J Clin Endocrinol Metab 2014;99:3635-43.

13. Dow C, Mancini F, Rajaobelina K, et al. Diet and risk of diabetic retinopathy: a systematic review. Eur J Epidemiol 2018;33:141-56.

14. Wong MYZ, Man REK, Fenwick EK, et al. Dietary intake and diabetic retinopathy: a systematic review. PLoS One 2018;13:e0186582.

15. Lee CT, Gayton EL, Beulens JW, et al. Micronutrients and diabetic retinopathy a systematic review. Ophthalmology 2010;117:71-8.

16. Shamseer L, Moher D, Clarke M, et al. Preferred reporting items for systematic review and meta-analysis protocols (PRISMA-P) 2015: elaboration and explanation. BMJ 2015;350:g7647.

17. Kennedy ET, Ohls J, Carlson S, et al. The Healthy Eating Index: design and applications. J Am Diet Assoc 1995;95:1103-8.

18. Guenther PM, Casavale KO, Reedy J, et al. Update of the Healthy Eating Index: HEl-2010. J Acad Nutr Diet 2013;113:569-80.
19. Appel LJ, Moore TJ, Obarzanek E, et al. A clinical trial of the effects of dietary patterns on blood pressure. DASH Collaborative Research Group. N Engl J Med 1997;336:1117-24.

20. Trichopoulou A, Kouris-Blazos A, Wahlqvist ML, et al. Diet and overall survival in elderly people. BMJ 1995;311:1457-60.

21. Sterne JA, Hernán MA, Reeves BC, et al. ROBINS-I: a tool for assessing risk of bias in non-randomised studies of interventions. BMJ 2016;355:i4919.

22. Schünemann HJ, Cuello $\mathrm{C}, \mathrm{Akl} \mathrm{EA}$, et al. GRADE guidelines: 18. How ROBINS-I and other tools to assess risk of bias in nonrandomized studies should be used to rate the certainty of a body of evidence. $J$ Clin Epidemiol 2018.

23. DerSimonian R, Laird N. Meta-analysis in clinical trials revisited. Contemp Clin Trials 2015;45(Pt A):139-45.

24. Greenland S, Longnecker MP. Methods for trend estimation from summarized dose-response data, with applications to meta-analysis. Am J Epidemiol 1992;135:1301-9.

25. Orsini N, Li R, Wolk A, et al. Meta-analysis for linear and nonlinear dose-response relations: examples, an evaluation of approximations, and software. Am J Epidemiol 2012;175:66-73.

26. Aune D, Greenwood DC, Chan DS, et al. Body mass index, abdominal fatness and pancreatic cancer risk: a systematic review and non-linear dose-response meta-analysis of prospective studies. Ann Oncol 2012;23:843-52.

27. Borenstein M, Higgins JP, Hedges LV, et al. Basics of meta-analysis: $\mathrm{I}^{2}$ is not an absolute measure of heterogeneity. Res Synth Methods 2017;8:5-18.

28. IntHout J, loannidis JP, Rovers MM, et al. Plea for routinely presenting prediction intervals in meta-analysis. BMJ Open 2016;6:e010247.

29. Stroup DF, Berlin JA, Morton SC, et al. Meta-analysis of observational studies in epidemiology: a proposal for reporting. Meta-analysis Of Observational Studies in Epidemiology (MOOSE) group. JAMA 2000;283:2008-12.

30. Bücker B, Wilm S, Arnolds S, et al. What are the preferences of people with diabetes for future diabetes research? - A qualitative study. Diabetologie und Stoffwechsel 2019;14:56-63. 